\title{
Confined three-dimensional stability analysis of the cylinder wake
}

\author{
Dwight Barkley* \\ Mathematics Institute, University of Warwick, \\ Coventry CV4 7AL, United Kingdom
}

(Dated: August 15, 2018)

\begin{abstract}
A spatially confined stability analysis is reported for the cylinder wake at Reynolds numbers 190 and 260. The two three-dimensional instabilities at these Reynolds numbers are shown to be driven by the flow just behind the cylinder.
\end{abstract}


Consider the von Kármán vortex street generated by flow past an infinitely long circular cylinder. This flow is time periodic and two dimensional for Reynolds numbers between approximately 47 and 189 [1, 2, 3, 44]. (The Reynolds number is $R e \equiv U_{\infty} d / \nu$, where $U_{\infty}$ is the fluid velocity far from the cylinder, $d$ is the cylinder diameter, and $\nu$ is the kinematic viscosity.) At Reynolds number 189 the $2 \mathrm{D}$ vortex street becomes three dimensionally unstable 3,4 , 5, 6, 7]. A numerical stability analysis of the flow up to Reynolds number 300 has determined two separate bands of linearly unstable modes [5]. The first appears at $R e=189$ with a spanwise wavelength of 4 cylinder diameters and the second appears at $R e=260$ with a spanwise wavelength of 0.8 diameters. These linear instabilities are related to 3D shedding modes known as mode A and mode B, first observed experimentally by Williamson [3].

In this paper I revisit the stability analysis of the vortex street. I show that small regions of the full flow just behind the cylinder are responsible for the 3D linear instabilities despite the fact that the actual linear modes extend many cylinder diameters downstream of the cylinder. This is important for two separate reasons. The first is that this limits the regions which should be analyzed either to clarify instability mechanisms or to suppress the instabilities if so desired. The second reason is that the stability analysis of small flow regions is computationally very efficient compared with a stability analysis of the full flow field. Thus this approach provides a method for quickly obtaining approximate stability information about a complex flow.

The computational approach is described fully elsewhere [5]. I summarize briefly the main points with focus on the important aspects for the current work. First direct simulations of the incompressible Navier-Stokes equations are used to obtain 2D, time-periodic wake flows: $\mathbf{U}(x, y, t+T)=\mathbf{U}(x, y, t)$ where $T$ is the wake period. A spectral-element method is employed on a computational domain shown in Fig. 1. The length scale is the cylinder diameter. The size of the computational domain depends on the Reynolds number and the accuracy required [5]. The domain shown gives sufficiently accurate results for the purposes of this work.

The boundary conditions imposed for the computation of $\mathbf{U}$ are as follows. On the top, bottom, and left boundaries uniform flow is imposed $\mathbf{U}=(U, V)=(1,0)$. At the right edge 

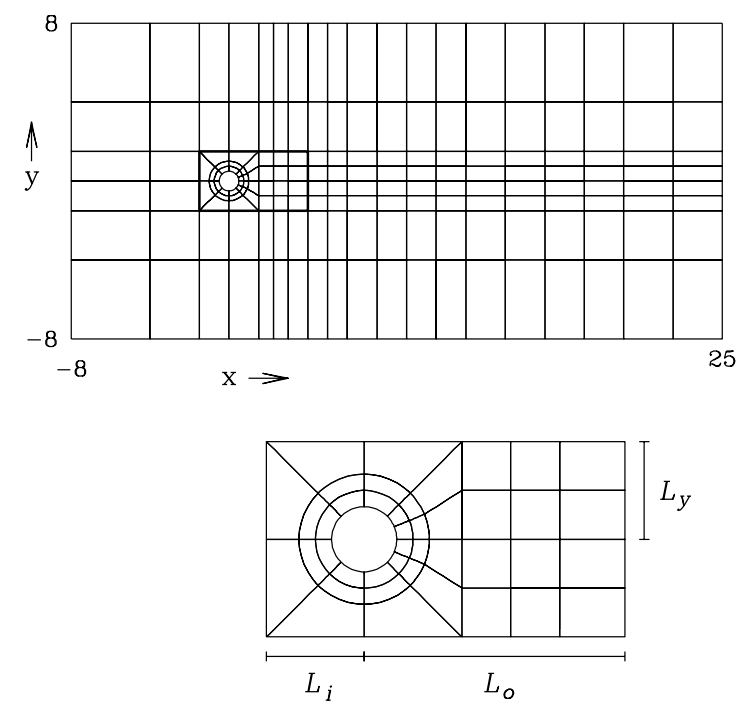

FIG. 1: Spectral-element computational mesh. The base flow is computed on the full domain and stability computations are performed on the full domain and on a variety of subdomains. The subdomain outlined in bold and shown as an enlargement has inflow length $L_{i}=1.5$, outflow length $L_{o}=4$, and cross-stream height $L_{y}=1.5$.

a (Neumann) outflow boundary condition for the velocity and pressure $P$ is used:

$$
\partial U / \partial x=0, \quad \partial V / \partial x=0, \quad P=0 .
$$

No-slip conditions are imposed at the cylinder surface.

The next step is a Floquet stability analysis of $3 \mathrm{D}$ disturbances to the $2 \mathrm{D}$ wake. This analysis is based on the evolution of infinitesimal 3D perturbations $\mathbf{u}^{\prime}(x, y, z, t)$ of the $2 \mathrm{D}$ flow $\mathbf{U}(x, y, t)$. The equation for such a perturbation is obtained by linearizing the Navier-Stokes equations about $\mathbf{U}$ :

$$
\frac{\partial \mathbf{u}^{\prime}}{\partial t}=-(\mathbf{U} \cdot \nabla) \mathbf{u}^{\prime}-\left(\mathbf{u}^{\prime} \cdot \nabla\right) \mathbf{U}-\frac{1}{\rho} \nabla p^{\prime}+\frac{1}{R e} \nabla^{2} \mathbf{u}^{\prime},
$$

where $p^{\prime}$ is the perturbation to the pressure that enforces $\nabla \cdot \mathbf{u}^{\prime}=0$.

In this work I shall primarily consider Eq. (2) posed on subdomains such as that illustrated in Fig. 1. These subdomains are characterized by their inflow and outflow lengths $L_{i}$ and $L_{o}$, measured with respect to the cylinder center, and their cross-flow range $-L_{y} \leq y \leq L_{y}$. The base flow $\mathbf{U}$ appearing in Eq. (2) is obtained by restricting the base flow from the full domain to the appropriate subdomain. 
Boundary conditions on $\mathbf{u}^{\prime}$ are required by Eq. (2). Consider the standard case in which $\mathbf{u}^{\prime}$ is computed on the same domain as $\mathbf{U}$. Then the requirement that $\mathbf{U}+\mathbf{u}^{\prime}$ satisfy the same boundary conditions as $\mathbf{U}$ gives homogeneous boundary conditions on $\mathbf{u}^{\prime}$ : $\mathbf{u}^{\prime}=(0,0,0)$ on all domain boundaries except the outflow boundary where $\mathbf{u}^{\prime}$ satisfies the analogy of Eq. (11). One can view these boundary conditions as the requirement that the perturbation be zero at the inflow and lateral boundaries of the domain but that perturbations can advect out of the domain. Based on this reasoning we impose these homogeneous boundary conditions when computing $\mathbf{u}^{\prime}$ on subdomains. We are interested in examining perturbations $\mathbf{u}^{\prime}$ which are zero on all boundaries of the subdomain except the outflow boundary. Perturbations are allowed to advect out of the subdomain.

From the linearized equations the spectrum of Floquet multipliers $\mu$ and corresponding Floquet modes $\tilde{\mathbf{u}}$ can be found [5]. Exponentially growing perturbations correspond to multipliers outside the unit circle in the complex plane $(|\mu|>1)$. Because the geometry is homogeneous in the spanwise direction, Floquet modes decouple in spanwise wavenumber $\beta$. The Floquet multipliers are thus computed as a function of $\beta$ on a number of subdomains for $R e=190$ and $R e=260$, just above the critical Reynolds numbers for the 3D instabilities.

Figure 2 summarizes the study in terms of multiplier spectra while Figs. 314 shows representative Floquet modes. A large number of subdomains have been studied but only representative cases are shown near the minimum dimensions necessary to capture the 3D instabilities.

Figure 2 primarily demonstrates the effect of outflow length $L_{o}$ on the Floquet multiplier spectrum. The inflow length and cross-stream height are fixed at minimal values of $L_{i}=0$ and $L_{y}=1.5$. One sees that $L_{o}=3$ is sufficient to capture the destabilizing Floquet mode. Increasing $L_{o}$ further has minimal effect. For $R e=190$, decreasing $L_{o}$ to 2.25 causes a substantial change to the spectrum - the subdomain is evidently too small to capture all the relevant flow features which drive the instability. In the case of $R e=260$, decreasing $L_{o}$ to 2.25 causes only a small change to the spectrum and the subdomain is essentially still large enough to capture relevant flow features. Decreasing the outflow length to $L_{o}=1.5$ causes the spectrum in both cases to deviate wildly from the correct behavior (at $R e=260$ the multipliers become complex with large magnitude and cannot be plotted on the scale of Fig. (2). Figs. 3]4 shows just how well the Floquet modes are captured by the subdomain computations even though the Floquet mode on the full domain extends far downstream of 


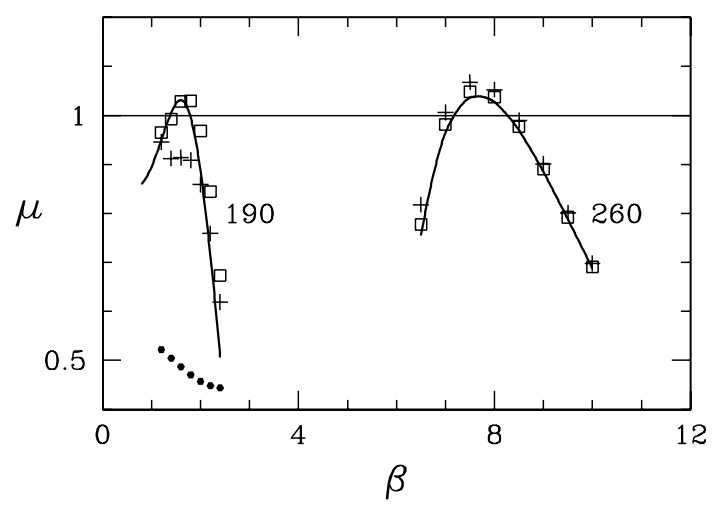

FIG. 2: Summary of Floquet multipliers at $R e=190$ and $R e=260$ (as labeled) for $\beta$ near the critical value in each case. Solid curves are from computations on the full domain. Points are from subdomains with $L_{i}=0, L_{y}=1.5$, and $L_{o}=3$ (squares), $L_{o}=2.25$ (crosses), and $L_{o}=1.5$ (dots). The multipliers for $R e=260, L_{o}=1.5$ are off the scale of the figure.

the cylinder [5].

Increasing the inflow length $L_{i}$ has some effect on the spectrum of multipliers in the case of $R e=190$. However, the effect is very small at the critical wavenumber where the multiplier branch has largest magnitude. Increasing the inflow length has almost no effect in the case $R e=260$. Increasing the cross-stream height has very little effect in either case. It is possible, in fact, that the cross-stream height could be reduced slightly from that used here without compromising the accuracy of the multipliers.

It is reasonable to conclude that the physical region responsible for the instability is localized within the subdomain in those cases where the Floquet multipliers and Floquet modes from subdomains are close to those from the full domain. This localization of the instability is similar to the transition from steady flow to vortex shedding which takes place at the primary wake instability. In that case there is a local, absolutely unstable region extending approximately three to four diameters downstream [ㅇ, 9$]$, very similar to the scales observed here. In fact the current computational analysis is similar in spirit to that of Hannemann and Oertel [8] and can be thought of as situated between the local analysis of a parallel wake profile and the global analysis previously reported for the secondary instability to three dimensionality [5].

Finally, it is worth considering the stability of the downstream portion of the flow. In 


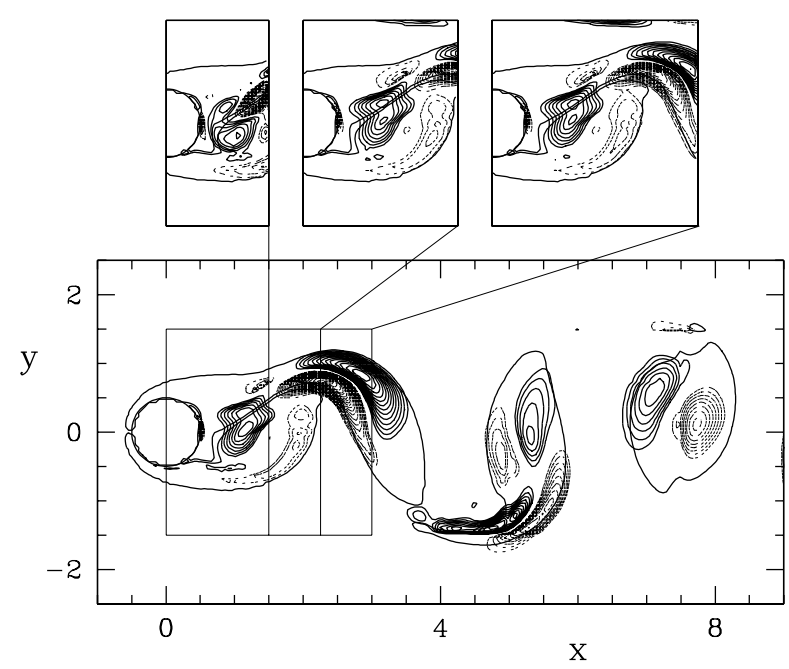

FIG. 3: Floquet modes at $R e=190$ with $\beta=1.6$. The main figure shows (a portion of) the mode computed on the full domain. Insets show modes computed on the subdomains indicated. (The inflow boundary $L_{i}=0$ and the lateral boundaries $L_{y}=1.5$ are the same for all subdomains.) All modes are plotted as spanwise vorticity contours at the same fixed time. The spanwise vorticity contours \pm 0.4 of the base flow are also plotted.

this case the base flow is projected onto the subdomain with $2.25 \leq x \leq 25$ and $-4 \leq y \leq 4$. Both at $R e=190$ and $R e=260$ the flow in this downstream region is found to be very stable. The maximum modulus of the Floquet multipliers are $|\mu| \simeq 0.28$ at $R e=190$, $\beta=1.6$ and $|\mu| \simeq 0.19$ at $R e=260, \beta=7.5$.

In this paper I have shown how a local Floquet stability analysis of small regions of the wake just behind a cylinder are sufficient to capture both 3D instabilities observed in wake transition. The specific results are of interest for future attempts to understand better the physical mechanisms of 3D transition [7, 10, 11, 12, 13] because the results set limits to the regions which could drive the instabilities. The general method is of interest for future computational studies of flow instabilities.

Much of this work was performed while visiting the Courant Institute of Mathematical Sciences, NYU. I thank members of the Institute for their hospitality.

* barkley@maths.warwick.ac.uk www.maths.warwick.ac.uk/ barkley 


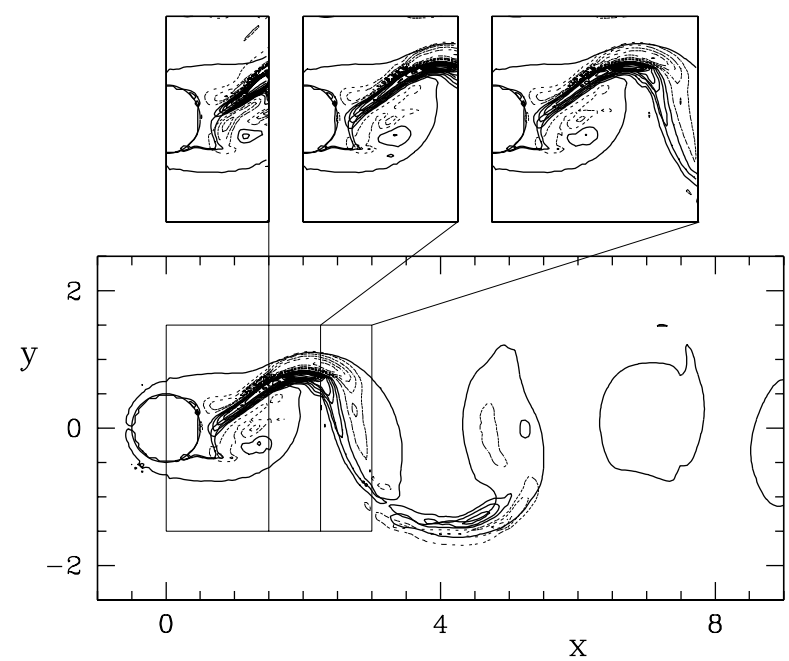

FIG. 4: Floquet modes at $R e=260$ with $\beta=7.7$ using the same conventions as Fig. 3

[1] C. P. Jackson, J. Fluid Mech. 182, 23 (1987).

[2] C. Mathis, M. Provansal, and L. Boyer, J. Fluid Mech. 182, 1 (1987).

[3] C. H. K. Williamson, Phys. Fluids 31, 3165 (1988).

[4] G. D. Miller and C. H. K. Williamson, Exp. Fluid. 18, 26 (1994).

[5] D. Barkley and R. D. Henderson, J. Fluid Mech. 322, 215 (1996).

[6] R. D. Henderson and D. Barkley, Phys. Fluids 8, 1683 (1996).

[7] C. H. K. Williamson, Ann. Rev. Fluid Mech. 28, 477 (1996).

[8] K. Hannemann and H. Oertel, J. Fluid Mech. 199, 55 (1989).

[9] H. Oertel, Ann. Rev. Fluid Mech. 22, 539 (1990).

[10] M. Brede, H. Eckelmann, and D. Rockwell, Phys. Fluids 8, 2117 (1996).

[11] H. Persillon and M. Braza, J. Fluid Mech. 365, 23 (1998).

[12] T. Leweke and C. H. K. Williamson, Eur. J. Mech. B/Fluids 17, 571 (1998).

[13] M. C. Thompson, T. Leweke, and C. H. K. Williamson, J. Fluid Struct. 15, 607 (2001). 